\title{
Isolating the Benefits of Respect
}

\author{
Stephen Chen \\ Information Technology \\ York University \\ 1-416-736-2100 x:30526 \\ sychen@yorku.ca
}

\author{
Gregory Pitt \\ Mathematics and Statistics \\ York university
}

greg@pittresearch.com

\begin{abstract}
The three mechanisms of crossover are transmission, assortment, and respect. Of these three mechanisms, assortment (i.e. recombination) is traditionally viewed as the primary feature and key advantage of crossover. However, respect (the preservation of common components) is also a feature that is unique to multiparent operators like crossover - it takes two (or more) parents to have/identify common components. The effects of respect are isolated from all other aspects of genetic algorithms by using a parallel implementation of simulated annealing. In this implementation, the preservation of common components is used to focus the search process and this focus has improved the performance of simulated annealing on the TSP. Since only the mechanism of respect is transferred from genetic algorithms to simulated annealing, these experiments isolate and demonstrate the benefits of respect.
\end{abstract}

\section{Categories and Subject Descriptors}

I.2.8 [Artificial Intelligence]: Problem Solving, Control Methods, and Search - heuristic methods.

\section{General Terms}

Algorithms, Theory.

\section{Keywords}

Heuristic Search, Genetic Algorithms, Simulated Annealing, Combinatorial Optimization, Commonality Hypothesis

\section{INTRODUCTION}

The mechanisms of crossover include "respect", "transmission", and "assortment" [4]. To legitimize respect as a design principle for crossover, it is important to demonstrate that its advantages (e.g. reduced implicit mutation) outweigh its disadvantages (e.g. premature convergence). Previous attempts to make this demonstration have failed to sufficiently isolate the effects of preserving common components from the remaining aspects of genetic algorithms (e.g. [3][4]). As a result, the improved performance of these GAs has not been clearly attributable to the mechanism of respect.

Copyright is held by the author/owner(s). GECCO'05, June 25-29, 2005, Washington, DC, USA. ACM 1-59593-010-8/05/0006.
To isolate the effects of respect, this feature has been transferred into simulated annealing (SA). The resulting system which combines features from simulated annealing and genetic algorithms is called SAGA. The results for SAGA are compared with the results from a similar, non-parallel implementation of simulated annealing for the TSP. Using the same number of twoopt swaps for both, the performance of SAGA is consistently and significantly better. These experiments isolate the effects of preserving common components, and the results demonstrate that respect is a beneficial mechanism that can be employed by multiparent operators. Therefore, respect should be viewed as an important design principle for the development of crossover and other multi-parent operators.

\section{SAGA}

SAGA starts with the control strategy for simulated annealing which is designed to allow probabilistic escapes from local optima. Assuming a minimization objective, the simulated annealing process can be visualized as a ball rolling downhill through a landscape of peaks and valleys. Depending on how much "energy" is in the ball, it has the ability to "bounce out" of local minima. When the temperature/energy approaches zero, the ball will come to rest in a final minimum - ideally the global minimum if the cooling schedule has been slow enough.

This control strategy does not specify how the "ball" will escape from local minima - it can climb any valley wall with equal probability. If local optima are randomly distributed throughout the search space, then this standard implementation of SA will be well suited. However, the local optima for many combinatorial optimization problems exhibit a "big valley" clustering - random local optima are more similar than random solutions, the similarities among local optima increase with their quality, and the global optimum is in the "centre" of the cluster of local optima [1].

The standard control strategy for simulated annealing is not as well suited for problems with "big valley" fitness landscapes. When escaping from local minima, simulated annealing makes no attempt to determine if it is climbing an "interior" wall (towards better local optima and the global optimum) or an "exterior" wall (towards inferior local optima). (See figure 1.) Although the proof of convergence for simulated annealing does not require this insight, the practical (time-based) performance of an SA implementation may be affected. 


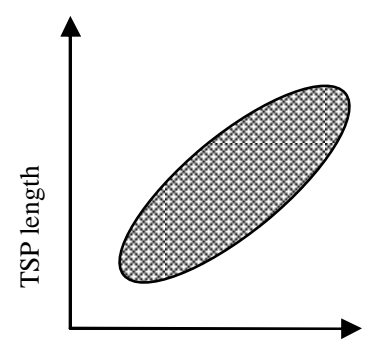

different edges

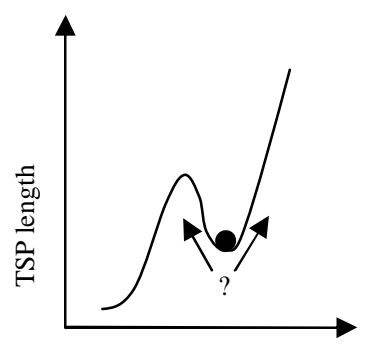

different edges
Fig. 1. In a typical distribution of local minima for the TSP, the best solutions have more common edges [1]. When escaping from a local minimum in simulated annealing, a change that produces additional different/uncommon edges may be moving away from the best solutions

In problems with big-valley fitness landscapes, changing a previously common component may increase the number of different edges and lead the search process to climb an exterior wall. Therefore, SAGA will use a "respectful" search operator to help direct the search process towards the centre of the big valley. To add the mechanism of respect to simulated annealing, multiple solutions are required. SAGA provides these solutions by using two parallel SA runs and storing the best solution after each "annealing stage".

SAGA has been developed for the TSP by using two parallel runs of a base SA application for the TSP (BaseSA) [2]. A two-opt swap changes two edges in a solution. To implement the mechanism of respect so that common components can be preserved, SAGA records the common edges of the two parallel BaseSA runs at the beginning of each annealing stage. The respectful two-opt operator will then select one uncommon edge and one random edge. This preference to change uncommon components will help preserve common components which should help direct the overall search process towards the centre of the "big valley" of local minima.

\section{EXPERIMENTS AND DISCUSSION}

Thirty trials of BaseSA and SAGA were run on each of the 5 test TSP instances. The results for the experiments are shown in table 1. The respectful two-opt operator in SAGA has led to consistent and significant improvements compared to the benchmark implementation of BaseSA. Since the performance of BaseSA is a controlled parameter in these experiments, any strengths or weaknesses in BaseSA should be reflected equally in the performance of both BaseSA and SAGA. Therefore, the superior performance of SAGA as compared to BaseSA is a clear demonstration of the benefits of respect.

Other researchers have attempted to combine the benefits of genetic algorithms and simulated annealing by using (only) recombination to coordinate parallel SA runs. These implementations have had mixed results and produced comments like "crossover can be compared to the very unpromising effort to recombine animals of different species" [5]. However, medical experiments for humans are often done on animals that we would not likely receive any benefit if we were to recombine with them (e.g. rats, monkeys, etc). These experiments exploit our common features with these animals, and SAGA exploits the common components of two solutions to coordinate parallel SA runs.

Table 1. Average percentage above optimal for 30 trials of BaseSA and SAGA with one billion total two-opt swaps performed. One-tailed t-tests show that the chance of the SAGA results being the same as the BaseSA results is less than $0.01 \%$ for all five instances

\begin{tabular}{lcccc}
\hline \multirow{2}{*}{ Instance } & \multicolumn{2}{c}{ BaseSA } & \multicolumn{2}{c}{ SAGA } \\
& avg. & std. dev. & avg. & std. dev. \\
\hline dsj1000 & 4.54 & 0.74 & 2.27 & 0.39 \\
d1291 & 8.87 & 1.41 & 3.12 & 1.12 \\
f11400 & 3.07 & 1.04 & 2.00 & 0.88 \\
f11577 & 6.47 & 1.58 & 0.64 & 0.55 \\
pr2392 & 9.24 & 1.37 & 6.53 & 0.56 \\
\hline
\end{tabular}

\section{CONCLUSIONS}

The three mechanisms of crossover have been presented as respect, transmission, and assortment. However, respect (the preservation and accumulation of common components) is also associated with negative aspects such as premature convergence and genetic drift. The experiments with SAGA isolate and demonstrate the benefits of respect - another feature that gives crossover operators and genetic algorithms an advantage over single-parent operators and point search techniques.

\section{ACKNOWLEDGMENTS}

The authors have received funding support from the Natural Sciences and Engineering Research Council of Canada and from the Atkinson Faculty of Liberal and Professional Studies, York University.

\section{REFERENCES}

[1] Boese, K.D.: Models for Iterative Global Optimization. Ph.D. diss., Computer Science Department, University of California at Los Angeles (1996)

[2] Chen, S., Pitt G.: The Coordination of Parallel Search with Common Components. To appear in Lecture Notes in Artificial Intelligence, Vol. TBA : Proceedings of the 18th International Conference on Industrial and Engineering Applications of Artificial Intelligence and Expert Systems (2005)

[3] Chen, S., Smith, S.F.: Introducing a New Advantage of Crossover: Commonality-Based Selection. In GECCO-99: Proceedings of the Genetic and Evolutionary Computation Conference. Morgan Kaufmann (1999)

[4] Radcliffe, N.J.: Forma Analysis and Random Respectful Recombination. In Proceedings of the Fourth International Conference on Genetic Algorithms. Morgan Kaufmann (1991)

[5] Wendt, O., König, W.: Cooperative Simulated Annealing: How much cooperation is enough? Technical Report, No. 1997-3, School of Information Systems and Information Economics at Frankfurt University (1997) 